\title{
Mineralogy and chemical composition of technogenic soils (Technosols) developed from fly ash and bottom ash from selected thermal power stations in Poland
}

\begin{abstract}
The aim of the study was to determine the mineral and chemical composition of technogenic soils (Technosols) developed from fly ash and bottom ash from power plants in which bituminous coal and lignite was combusted. The mineral composition of the "fresh" wastes (i.e. fly ash and bottom ash) and soil samples derived from them was examined by X-ray diffraction (XRD) and using a scanning electron microscope (SEM). The chemical composition (content of major elements) was determined using ICP-AES method. Quartz, mullite, and amorphous substances (glass) predominated in the mineral composition of wastes after bituminous coal combustion. Magnetite was also found there. Soils developed from wastes after bituminous coal combustion contained all above mentioned minerals inherited from fly ash and bottom ash. Moreover, small amounts of secondary calcite were identified. In some soil horizons containing large amounts of inherited magnetite, secondary iron oxides and oxyhydroxides (goethite and lepidocrocite) also occurred. Quartz predominated in the mineral composition of the "fresh" wastes after lignite combustion. Relatively small amounts of iron oxides (magnetite and hematite) were also found there. In "fresh" fly ash, apart from minerals mentioned above, anhydrite and calcium oxide (lime) was identified. Soils developed from wastes after lignite combustion contained inherited quartz, magnetite, and hematite. Furthermore, calcite which sometimes was a predominating mineral in certain soil horizons occurred. Moreover, sulphates (gypsum, bassanite, and ettringite), and vaterite (a polymorph of Ca carbonate) were also found in soils. Silicon predominated among major elements in "fresh" ashes after bituminous coal combustion and soil derived from them followed by Al, $\mathrm{Fe}, \mathrm{K}, \mathrm{Ca}, \mathrm{Mg}$, Ti, Na, P, and Mn. On the other hand, the contents of major elements in the samples (ashes and soils) after lignite combustion can be arranged as follows: $\mathrm{Si}, \mathrm{Ca}, \mathrm{Fe}, \mathrm{Al}, \mathrm{Mg}, \mathrm{Ti}, \mathrm{K}, \mathrm{Mn}, \mathrm{Na}$, and P. However, in some horizons (i.e. in calcareous materials deposited in the topsoil of some profiles) in soil developed on landfills near TPSs combusting lignite, Ca was a predominating element.
\end{abstract}

Keywords: fly ash, bottom ash, Technosols, X-ray diffraction, scanning electron microscopy, major elements

\section{INTRODUCTION}

Technogenic soils (Technosols) defined as soils strongly influenced by human (IUSS Working Group WRB 2015), develop, among others, from diverse industrial wastes. One of them are fly ash and bottom ash produced as a result of bituminous coal and lignite combustion in thermal power stations (TPSs). These wastes, despite their anthropogenic origin, undergo natural weathering after deposition on land surface in the soil environment.

One of the ways to determine the directions of weathering in soils developed from ashes are studies of mineral and chemical transformations occurring in soils (Maciak et al. 1976, Warren and Dudas 1985, Zikeli et al. 2002, Yeheyis et al. 2009). The key issue

The paper was presented at the 29. Congress of the Polish Society of Soil Science, Wrocław, Poland, $31^{\text {st }}$ August $-5^{\text {th }}$ September 2015. in recognition of these transformation is to know mineral and chemical composition of "fresh" fly ash and bottom ash. The ashes usually contain high amounts of amorphous glass consisting of aluminosilicate and ferric amorphous phases (e.g. Koukouzas et al. 2006), which generally form characteristic spherical grains with diameters about several dozen of micrometers (Sokol et al. 2000, 2002; Kutchko and Kim 2006). The main well-crystallized phases occurring in fly ash are minerals inherited from coal (e.g. quartz, feldspars, kaolinite, carbonates), high-temperature mineral phases which originate during combustion at the expense of inherited minerals (e.g. mullite, gehlenite, and portlandite), sulphates (mainly gypsum) which are the products of oxidation of sulphides occurring in coal, as well as magnetic minerals (mainly magnetite) which may be inherited from coal or may represent secondary high-temperature phases (e.g. Koukouzas et al. 2006). 
Coal combustion wastes (fly and bottom ashes) after the deposition on land surface and development of soils on them undergo mineral transformations. The previous studies showed, for example, that during the development of soils on fly ash landfills the decrease of amount of gypsum in the uppermost parts of the soils proceeds, as the mineral is leached out from the soil (Zikeli et al. 2002). The investigations also proved, that calcium carbonates develop in soil profiles as a result of the reaction of calcium oxides occurring in fly ash with carbon dioxide from the atmosphere (Maciak et al. 1976, Zikeli et al. 2002, Yeheyis et al. 2009). Carbonates are leached from the upper parts of the profiles as the weathering proceeds (Warren and Dudas 1985).

The objective of the present study was to determine the general mineralogy and chemical composition (contents of major elements) of technogenic soils (Technosols) developed from fly ash and bottom ash from selected TPSs in Poland. These preliminary studies allowed the authors to give the idea about the mineral transformations in the soils studied.

\section{MATERIALS AND METHODS}

Soil profiles developed from lignite and bituminous coal combustion wastes were investigated. They were located on landfills of three thermal power stations in Poland: (1) the "Laziska" TPS (bituminous coal) located in Łaziska Górne town, and (2) the "Pątnów" and "Konin" TPSs (lignite) located in Konin town. Moreover, the samples of "fresh", unweathered wastes (fly ash and bottom ash) taken from power stations were also analysed in order to compare their mineral and chemical composition with soil samples.

Soil samples were taken from each soil horizon (layer) distinguished during the field works. Soil and "fresh" waste samples were air dried and sieved $(<2 \mathrm{~mm})$. Physical and chemical properties of the fine earth material $(<2 \mathrm{~mm})$ were determined using common pedological methods (Pansu and Gautheyrou 2006).

The waste and soil samples were ground in the zirconium mortar and powders were analysed using $\mathrm{X}$-ray diffraction method (XRD) with the use of Bruker AXS D5005 diffractometer equipped with the KRISTALLOFLEX ${ }^{\circledR} 760 \mathrm{X}$-ray generator, the vertical goniometer, $1 \mathrm{~mm}$ divergence slit, $2 \mathrm{~mm}$ anti scatter slit, 0.6 detector slit, and a graphite diffracted-beam monochromator. CoK $\alpha$ radiation was used with the applied voltage of $40 \mathrm{kV}$ and $30 \mathrm{~mA}$ current. Random mounts of the ground materials were scanned from 3 to $60^{\circ} 2 \theta$ at a counting time of $2 \mathrm{~s}$ per $0.01^{\circ}$ step. XRD analyses were performed in the Department of Soil Environment Sciences, Warsaw University of Life Sciences - SGGW, Poland.

The selected air-dry waste and soil samples (fraction $<2 \mathrm{~mm}$ ) were analysed using scanning electron microscope (SEM) in order to observe the morphology of the mineral phases in the microscale. The analyses were performed in the Analytical Centre, Warsaw University of Life Sciences - SGGW using FEI Quanta 200 SEM equipped with a tungsten filament cathode. The accelerating voltage of $20 \mathrm{kV}$ was used with a spot size of $3 \mu \mathrm{m}$.

In order to determine the contents of major elements, the samples were ground and mixed with $\mathrm{LiBO}_{2} / \mathrm{Li}_{2} \mathrm{~B}_{4} \mathrm{O}_{7}$ flux. Crucibles were fused in a furnace. The cooled beads were dissolved in the American Chemical Society (ACS) grade nitric acid and analyzed by ICP-AES method. Loss on ignition (LOI) was determined by igniting a sample split in $1000^{\circ} \mathrm{C}$, then measuring the weight loss. Contents of major elements and LOI were determined in the Bureau Veritas Minerals Laboratories (formerly ACME laboratories).

\section{RESULTS AND DISCUSSION}

\section{Description of the soils studied}

All soils investigated were weakly developed technogenic soils (Technosols). Profiles from the vicinity of the "Laziska" TPS (the label of soil profiles is "LA") were developed from wastes after bituminous coal combustion, and profiles from the vicinity of the "Pątnów" and "Konin" TPSs (the label of soil profiles is "KN") originated form fly ash and bottom ash after lignite combustion.

The analysis and interpretation of physical and chemical properties of soils was not the objective of the present paper, therefore they are only briefly presented below. The soils investigated were characterized by variation of texture within the soil profiles. The $\mathrm{pH}$ value measured in $1 \mathrm{~mol} \cdot \mathrm{dm}^{3} \mathrm{KCl}$ ranged from 5.2 and 10.4 (landfills at bituminous coal fired power plants) and from 8.9 to 12.1 (landfill at lignite fired power plants). Carbonate content was variable in soil profiles and amounted up to 4\% (landfills at bituminous coal fired power plants) and up to $82.7 \%$ (landfill at lignite fired power plants). The soils investigated contained variable amounts of organic carbon which the highest concentrations were found in the topsoils. Base cations ( $\mathrm{Ca}$ followed by $\mathrm{Mg}$ ) predominated in the sorption complex of the soils studied, which resulted in a high value of base saturation (generally more than $90 \%$ ). 


\section{Mineral composition of wastes and soils}

Quartz $\left(\mathrm{SiO}_{2}\right)$ and mullite $\left(\mathrm{Al}_{6} \mathrm{Si}_{2} \mathrm{O}_{13}\right)$ predominated in the mineral composition of the "fresh" fly ash and bottom ash derived after bituminous coal combustion (Table 1, Fig. 1A), which is a common feature for such wastes (e.g. Koukouzas et al. 2007, Kierczak and Chudy 2014). Amorphous substances (glass) were also abundant, which was corroborated by the presence of distinct hump around $25-30^{\circ} 2 \theta$ in the XRD patterns (Fig. 1A and $\mathrm{B}$ ). Iron oxides - mainly magnetite $\mathrm{Fe}_{3}\left(\mathrm{Fe}^{2+} \mathrm{Fe}^{3+}\right) \mathrm{O}_{4}$ - were also found there. Soils developed from wastes derived after bituminous coal combustion contained all above mentioned minerals inherited from fly ash and bottom ash (Table 1, Fig. 1B). Moreover, small amounts of calcite $\left(\mathrm{CaCO}_{3}\right)$ were identified (Table 1), which most likely crystallized from soil solution rich in calcium and $\mathrm{CO}_{2}$ (e.g. Kierczak and Chudy 2014). In some soil horizons containing large amounts of inherited magnetite (e.g. AC2 horizon in LA2 profile), secondary iron oxides and oxyhydroxides (goethite and lepidocrocite) also occurred (Table 1). The secondary iron oxides mentioned were most likely the effect of weathering in soil environment. Furthermore, kaolinite was found in the topsoil of LA4 profile (Table 1, Fig. 1B). This mineral can be either the effect of mineral transformations in soil environment or might be transported from the neighbouring bituminous coal mine dumps due to aeolian processes and deposited in the topsoil of the profile.

Mineral grains in soils developed from wastes after bituminous coal combustion occurred mostly in a form of spheroidal and oval-shape particles (Fig. 2). This type of shape is common in fly ash after bituminous coal combustion in which spherical grains with diameters about several dozen of micrometers consisting of aluminosilicate and ferric amorphous phases predominate (Sokol et al. 2000, 2002).

Quartz predominated in the mineral composition of the "fresh" fly ash and bottom ash after lignite combustion (Table 1, Fig. 1C). Relatively small amounts of iron oxides (magnetite and hematite $\alpha-\mathrm{Fe}_{2} \mathrm{O}_{3}$ ) were also found. In "fresh" fly ash, apart from minerals mentioned above, anhydrite $\left(\mathrm{CaSO}_{4}\right)$ and lime $(\mathrm{CaO})$ were identified (Fig. 1C). The occurrence of anhydrite seems to be a common feature for non-weathered fly ashes derived after lignite combustion (e.g. Zikeli et al. 2002), in particular for S-rich lignites. Moreover, traces of feldspars were found in ashes examined (Table 1).

* Minerals are arranged in a semiquantitative order based on the relation of peak intensities in the XRD patterns. Predominant minerals are in bold. Symbols of mineral phases: A - anhydrite, AS - amorphous substance (glass), B - bassanite, $\mathrm{Br}$ - brucite, $\mathrm{C}$ - calcite, $\mathrm{E}$ - ettringite, $\mathrm{F}$ - feldspar, Ge - goethite, Gy - gypsum, $\mathrm{H}$ - hematite, $\mathrm{K}$ - kaolinite, $\mathrm{L}$ - lime (Ca oxide), Le - lepidocrocite, $\mathrm{M}$ - magnetite, Mo - monohydrocalcite, $\mathrm{Mu}$ - mullite, $\mathrm{Q}$ - quartz, $\mathrm{V}$ - vaterite.
TABLE 1. Mineral composition of the fine earth $(<2 \mathrm{~mm})$ of "fresh" ashes and soils based on XRD analyses for powders

\begin{tabular}{|c|c|c|c|}
\hline Profile & Horizon & Depth $(\mathrm{cm})$ & Mineral phases* \\
\hline \multicolumn{3}{|c|}{ The "fresh" fly ash from the Łaziska TPS } & $\mathbf{A S}, \mathbf{Q}, \mathrm{Mu}, \mathrm{M}$ \\
\hline \multicolumn{3}{|c|}{$\begin{array}{l}\text { The "fresh" bottom ash from the } \\
\text { Łaziska TPS }\end{array}$} & $\mathbf{Q}, \mathrm{Mu}, \mathrm{AS}, \mathrm{M}, \mathrm{F}$ \\
\hline LA1 & $\begin{array}{l}\text { OeiC } \\
\text { AC } \\
\text { C } \\
2 \mathrm{C} \\
3 \mathrm{C} \\
4 \mathrm{C} \\
\end{array}$ & $\begin{array}{c}0-1 \\
1-5 \\
5-30 \\
30-70 \\
70-100 \\
110-125 \\
\end{array}$ & $\begin{array}{l}\text { not determined } \\
\mathbf{Q}, \mathbf{A S}, \mathrm{Mu}, \mathrm{C}, \mathrm{M} \\
\mathbf{Q}, \mathbf{A S}, \mathrm{Mu}, \mathrm{C}, \mathrm{M} \\
\mathbf{Q}, \mathbf{A S}, \mathrm{Mu}, \mathrm{C}, \mathrm{M} \\
\mathbf{Q}, \mathbf{M u}, \mathrm{AS}, \mathrm{C}, \mathbf{M} \\
\mathbf{Q}, \mathbf{A S}, \mathrm{Mu}, \mathrm{C}, \mathbf{M}\end{array}$ \\
\hline LA2 & $\begin{array}{l}\mathrm{OiC} \\
\mathrm{AC} 1 \\
\mathrm{AC} 2 \\
\mathrm{C} \\
2 \mathrm{C} \\
3 \mathrm{C} \\
4 \mathrm{C} \\
5 \mathrm{C} \\
6 \mathrm{C} \\
7 \mathrm{C} \\
8 \mathrm{C} \\
9 \mathrm{C} \\
10 \mathrm{C} \\
11 \mathrm{C} \\
12 \mathrm{C} \\
13 \mathrm{C} \\
\end{array}$ & $\begin{array}{c}0-1 \\
1-4 \\
4-6 \\
6-25 \\
25-45 \\
45-65 \\
65-68 \\
68-73 \\
73-80 \\
80-108 \\
108-110 \\
110-120 \\
120-128 \\
128-153 \\
153-165 \\
165-170 \\
\end{array}$ & $\begin{array}{l}\text { not determined } \\
\mathbf{Q}, \mathrm{Mu}, \mathrm{AS}, \mathrm{M}, \mathrm{H}, \mathrm{C} \\
\mathbf{M}, \mathbf{Q}, \mathrm{Mu}, \mathrm{AS}, \mathrm{Ge}, \mathrm{Le}, \mathrm{H} \\
\mathbf{Q}, \mathbf{M u}, \mathrm{AS}, \mathrm{M}, \mathrm{C} \\
\mathbf{Q}, \mathbf{M u} \text { AS, M } \\
\mathbf{Q}, \mathbf{M u} \text { AS, M } \\
\mathbf{Q}, \mathbf{M u}, \mathrm{AS}, \mathbf{M} \\
\mathbf{Q}, \mathbf{M u} \text { AS, M } \\
\mathbf{Q}, \mathbf{M u}, \mathrm{AS}, \mathrm{C} \\
\mathbf{Q}, \mathrm{Mu}, \mathrm{AS}, \mathrm{M}, \mathrm{C} \\
\mathbf{Q}, \mathbf{M u}, \mathrm{AS}, \mathrm{C} \\
\mathbf{Q}, \mathrm{C}, \mathrm{Mu}, \mathrm{AS} \\
\mathbf{Q}, \mathrm{Mu}, \mathrm{C}, \mathrm{AS} \\
\mathbf{Q}, \mathrm{Mu}, \mathrm{C}, \mathrm{AS}, \mathrm{M} \\
\mathbf{Q}, \mathrm{Mu}, \mathrm{AS} \\
\mathbf{Q}, \mathrm{Mu}, \mathrm{AS}\end{array}$ \\
\hline LA3 & $\begin{array}{l}\text { OeiC } \\
\text { OaeC } \\
\text { AC } \\
\text { C } \\
2 \mathrm{C} \\
3 \mathrm{C} \\
4 \mathrm{C} \\
5 \mathrm{C} \\
6 \mathrm{C} \\
7 \mathrm{C} \\
8 \mathrm{C} \\
9 \mathrm{C} \\
\end{array}$ & $\begin{array}{c}0-2 \\
2-6 \\
6-15 \\
15-37 \\
37-57 \\
57-67 \\
67-78 \\
78-90 \\
90-100 \\
100-107 \\
107-122 \\
122-130 \\
\end{array}$ & $\begin{array}{l}\text { Q, AS, Mu, M } \\
\text { Q, AS, Mu, M } \\
\text { Q, Mu, AS, M } \\
\text { Q, Mu, AS, M, C } \\
\text { Q, Mu, AS, M, C } \\
\text { Q, Mu, AS, M, C } \\
\text { Q, Mu, AS, M } \\
\text { Q, Mu, AS, M } \\
\text { Q, Mu, AS, M } \\
\text { Q, Mu, AS, F, M } \\
\text { Q, Mu, AS, C, M } \\
\text { Q, Mu, AS, M, C }\end{array}$ \\
\hline LA4 & $\begin{array}{l}\text { Oei } \\
\text { A1 } \\
\text { A2 } \\
\text { AC } \\
\text { C } \\
2 \mathrm{C} \\
3 \mathrm{C} \\
\end{array}$ & $\begin{array}{c}0-3 \\
3-10 \\
10-18 \\
18-30 \\
30-70 \\
70-115 \\
115-135 \\
\end{array}$ & $\begin{array}{l}\text { Q, AS, Mu, M } \\
\text { Q, Mu, AS, M, K, Ge } \\
\text { Q, Mu, AS, M } \\
\text { Q, Mu, AS, M, C } \\
\text { Q, Mu, AS, C, M } \\
\text { Q, Mu, AS, C, M } \\
\text { Q, Mu, AS, M, C } \\
\end{array}$ \\
\hline
\end{tabular}

The "fresh" fly ash from the Pąnów TPS Q, A, L, M, H, F?

\begin{tabular}{|c|c|c|c|}
\hline \multicolumn{3}{|c|}{$\begin{array}{l}\text { The "fresh" bottom ash from the } \\
\text { Pątnów TPS }\end{array}$} & \multirow{2}{*}{$\begin{array}{l}\text { Q, H, F? } \\
\mathbf{C}, \mathrm{Q}, \mathrm{F} ? \\
\mathbf{Q}, \mathrm{C}, \mathrm{M}, \mathrm{H}, \mathrm{F} ? \\
\mathbf{Q}, \mathrm{M}, \mathrm{H}, \mathrm{C} \\
\mathbf{Q}, \mathrm{C}, \mathrm{H}, \mathrm{M}, \mathrm{Gy}, \mathrm{B}, \mathrm{F} ? \\
\mathbf{Q}, \mathrm{M}, \mathrm{C}, \mathrm{H}\end{array}$} \\
\hline KN2 & $\begin{array}{l}\mathrm{C} \\
2 \mathrm{C} \\
3 \mathrm{C} \\
4 \mathrm{C} \\
5 \mathrm{C}\end{array}$ & $\begin{array}{c}0-16 \\
16-32 \\
32-50 \\
50-110 \\
110-115\end{array}$ & \\
\hline KN3 & $\begin{array}{l}\mathrm{OiC} \\
\mathrm{AC} \\
\mathrm{C} \\
2 \mathrm{C} \\
3 \mathrm{C} \\
4 \mathrm{C} \\
5 \mathrm{C}\end{array}$ & $\begin{array}{c}0-1 \\
1-3 \\
3-7 \\
7-12 \\
12-25 \\
25-50 \\
50-55\end{array}$ & $\begin{array}{l}\text { C, Q, Gy, H, M } \\
\text { C, Q, Gy, H, M } \\
\text { C, Q, M, H } \\
\text { Q, M, Gy, H, C } \\
\text { Q, M, H, Gy } \\
\text { Q, M, H, Gy } \\
\text { Q, C, M, Gy, H, E, B }\end{array}$ \\
\hline KN5 & $\begin{array}{l}\text { Oei } \\
\text { A } \\
\text { C } \\
2 \mathrm{C} \\
3 \mathrm{C} \\
4 \mathrm{C} \\
5 \mathrm{C}\end{array}$ & $\begin{array}{c}0-2 \\
2-7 \\
7-25 \\
25-60 \\
60-70 \\
70-76 \\
76-80\end{array}$ & $\begin{array}{l}\text { Q, C, H, F? } \\
\mathbf{Q}, \mathbf{C}, \mathrm{H} \\
\mathbf{C}, \mathrm{Q}, \mathrm{H} \\
\mathbf{C}, \mathbf{Q}, \mathrm{Gy}, \mathrm{Mo} \text { ? } \\
\mathbf{Q}, \mathbf{E}, \mathrm{V}, \mathrm{Gy}, \mathrm{C}, \mathrm{B}, \mathrm{Br}, \mathrm{Mo} \text { ? } \\
\mathbf{C}, \mathbf{Q}, \mathrm{E}, \mathrm{B}, \mathrm{V}, \mathrm{Gy} \\
\mathbf{Q}, \mathrm{V}, \mathrm{E}, \mathrm{Gy}, \mathrm{B}, \mathrm{C} \text { ?, Mo? }\end{array}$ \\
\hline
\end{tabular}




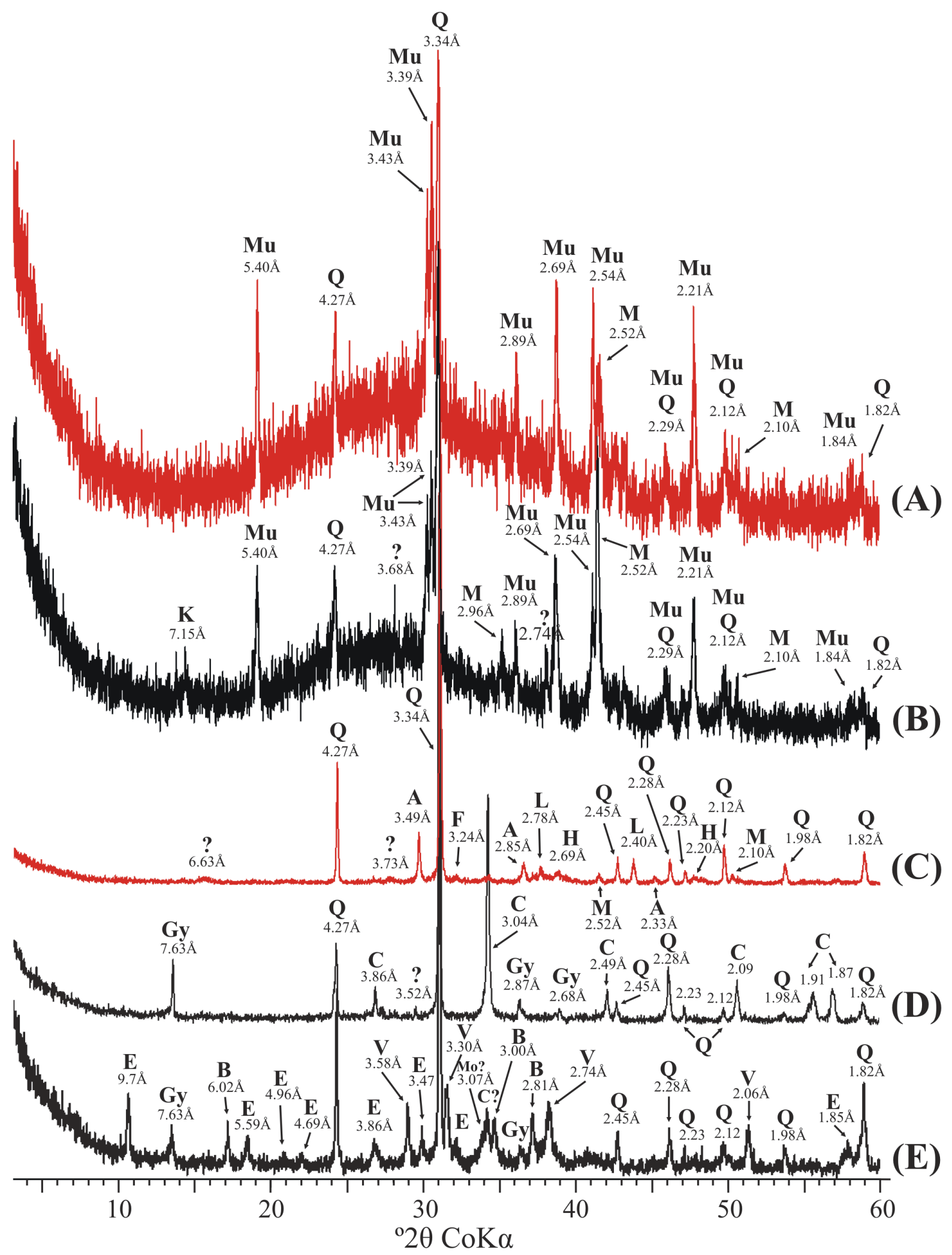

FIGURE 1. XRD patterns of "fresh" (unweathered) fly ashes and selected soil samples: (A) "fresh" fly ash after bituminous coal combustion, the Łaziska TPS, (B) soil sample from profile LA4, A1 horizon, (C) "fresh" fly ash after lignite combustion, the Pątnów TPS, (D) soil sample from KN5 profile, 2C horizon, and (E) soil sample from KN5 profile, 5C horizon. Symbols of minerals: A - anhydrite, B - bassanite, C - calcite, E - ettringite, F - feldspar, Gy - gypsum, $\mathrm{H}$ - hematite, $\mathrm{K}$ - kaolinite, $\mathrm{L}$ - lime (Ca oxide), $\mathrm{M}$ - magnetite, $\mathrm{Mo}$ - monohydrocalcite, $\mathrm{Mu}$ - mullite, $\mathrm{Q}$ - quartz, $\mathrm{V}$ - vaterite 
Soils developed from wastes after lignite combustion contained quartz, magnetite, and hematite inherited from fly ash and bottom ash (Table 1, Fig. 1D). Furthermore, calcite was sometimes a predominating mineral in certain soil horizons, in particular in calcareous materials deposited in topsoil of the KN2 and $\mathrm{KN} 3$ profiles, and some horizons in the $\mathrm{KN} 5$ profile (Table 1). Diverse amounts of gypsum were also found in the soils. Technogenic soils developed from wastes after lignite combustion contained some other sulphate minerals, e.g. ettringite $\mathrm{Ca}_{6} \mathrm{Al}_{2}\left(\mathrm{SO}_{4}\right)_{3}(\mathrm{OH})_{12} \cdot 26 \mathrm{H}_{2} \mathrm{O}$ and bassanite $\mathrm{CaSO}_{4} \cdot 0.5\left(\mathrm{H}_{2} \mathrm{O}\right)$ (Table 1). Euhedral and/or subhedral acicular crystals shown in Figure 3 are most likely ettringite. Such habit is typical of this mineral, which is a common secondary mineral phase e.g. in concretes (e.g. Thomas et al. 2008). Furthermore, vaterite - a polymorph of $\mathrm{CaCO}_{3}$ rarely occurring in the environment - was found in the subsoil of KN5 profile (Table 1, Fig. 1E). To the best author's knowledge, vaterite has never been documented earlier in soils developed from ashes derived after coal and lignite combustion. The origin of vaterite in the profiles studied is unclear. However, it could precipitate in strongly alkaline ponds where fly ash was deposited as a water suspension. Vaterite is supposed to form in alkaline environments supersaturated with calcium most likely with a participation of microorganisms (e.g. Rowlands and Webster 1971, Rodriguez-Navarro et al. 2007). Apart the minerals mentioned, the occurrence of brucite $\mathrm{Mg}(\mathrm{OH})_{2}$ (a main peak of 2.37 A, not shown in the XRD patterns) and monohydrocalcite $\mathrm{CaCO}_{3} \cdot \mathrm{H}_{2} \mathrm{O}$ (a hump of $3.07 \AA$ on the highangle side of the 3.03 A peak of calcite, Fig. 1E) is also likely in the lower part of the KN5 profile (Table 1).

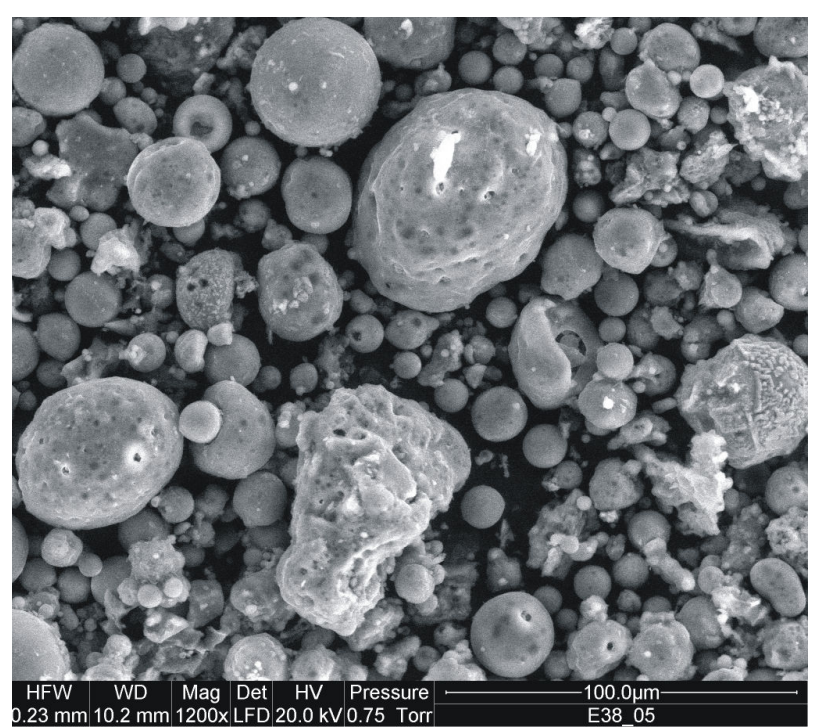

FIGURE 2. Morphology of mineral grains in soil developed from wastes after bituminous coal combustion (profile LA4, A2 horizon). SE image in SEM
Contents of major elements in wastes and soils

Contents of major elements in wastes (ashes) and soils under study were very diverse within the profiles. Moreover, there were distinct differences between chemical composition of samples after bituminous coal and lignite combustion.

Silicon and Al predominated in chemical composition of wastes and soils after bituminous coal combustion (Table 2). It is due to predominance of quartz, mullite, and amorphous substances (glass) rich in Si and $\mathrm{Al}$ in the mineral composition of these wastes and soils (Table 1). Contents of Si ranged from 27.11 to $53.32 \%(46.45 \%$ on average $)$, and the amounts of Al were between 11.23 and $28.10 \%$ (23.73\% on average). The wastes and soils after bituminous coal combustion contained $\mathrm{Fe}$, which amounts ranged from 3.80 to $43.93 \%$ ( $8.14 \%$ on average) (Table 2$)$. The highest contents of Fe were found in the samples enriched in iron oxides (primarily magnetite), e.g. in AC2 horizon, LA2 profile (Table 1). The elements which occurred in wastes and soils after bituminous coal combustion in relatively high amounts were K (1.08-3.16\%, $2.55 \%$ on average), $\mathrm{Ca}(1.04-3.77 \%, 2.23 \%$ on average), and $\mathrm{Mg}(1.02-2.58 \%, 1.98 \%$ on average). Calcium occurred in the soils studied in a form of Ca carbonates, mainly calcite (Table 1). Majority of $\mathrm{K}$ and $\mathrm{Mg}$ were most likely bound in amorphous substances (glass). The amounts of $\mathrm{Ti}$ were between 0.49 and $1.17 \%$ $(0.90 \%$ on average). Contents of $\mathrm{Na}$ ranged from 0.20 and $1.07 \%(0.46 \%$ on average $)$. The wastes and soils after bituminous coal combustion contained $\mathrm{P}$, which amounts were between 0.21 and $0.92 \%(0.39 \%$ on average). These wastes and soils also contained low

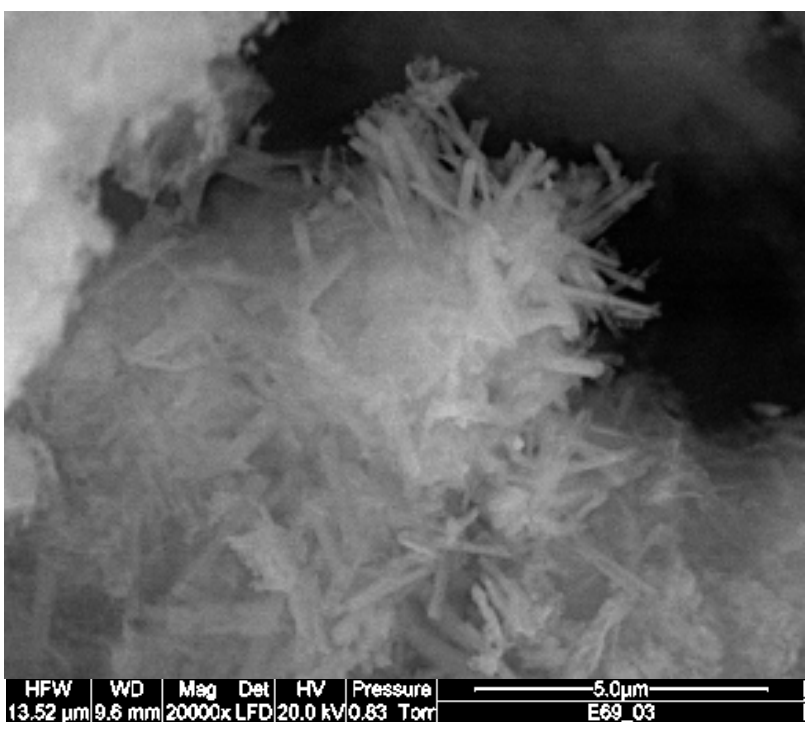

FIGURE 3. Acicular minerals, most likely ettringite, from soil profile developed from fly ash and bottom ash after lignite combustion (profile KN5, 3C horizon) SE image in SEM 
TABLE 2. Contents of major elements in the fine earth $(<2 \mathrm{~mm})$ of "fresh" ashes and soils studied (in \%)

\begin{tabular}{|c|c|c|c|c|c|c|c|c|c|c|c|c|c|c|}
\hline Profile & Horizon & Depth $(\mathrm{cm})$ & $\mathrm{SiO}_{2}$ & $\mathrm{Al}_{2} \mathrm{O}_{3}$ & $\mathrm{Fe}_{2} \mathrm{O}_{3}$ & $\mathrm{MgO}$ & $\mathrm{CaO}$ & $\mathrm{Na}_{2} \mathrm{O}$ & $\mathrm{K}_{2} \mathrm{O}$ & $\mathrm{TiO}_{2}$ & $\mathrm{P}_{2} \mathrm{O}_{5}$ & $\mathrm{MnO}$ & LOI & Sum \\
\hline \multicolumn{3}{|c|}{ The "fresh" fly ash from the Łaziska TPS } & 50.92 & 27.32 & 7.87 & 2.40 & 3.17 & 1.07 & 3.16 & 1.09 & 0.34 & 0.08 & 1.8 & 99.29 \\
\hline \multicolumn{3}{|c|}{ The "fresh" bottom ash from the Łaziska TPS } & 52.46 & 22.34 & 11.23 & 2.52 & 3.53 & 0.68 & 2.66 & 0.85 & 0.21 & 0.14 & 2.9 & 99.54 \\
\hline \multirow[t]{6}{*}{ LA1 } & OeiC & $0-1$ & 43.39 & 23.00 & 4.56 & 1.80 & 1.68 & 0.57 & 2.62 & 0.88 & 0.39 & 0.05 & 20.7 & 99.67 \\
\hline & $\mathrm{AC}$ & $1-5$ & 44.66 & 24.31 & 4.65 & 1.82 & 1.86 & 0.57 & 2.71 & 0.91 & 0.28 & 0.05 & 17.8 & 99.66 \\
\hline & $\mathrm{C}$ & $5-30$ & 44.88 & 24.23 & 4.71 & 1.86 & 1.92 & 0.61 & 2.68 & 0.91 & 0.30 & 0.05 & 17.5 & 99.67 \\
\hline & $2 \mathrm{C}$ & $30-70$ & 47.39 & 25.10 & 4.46 & 1.73 & 1.91 & 0.51 & 3.06 & 0.90 & 0.26 & 0.04 & 14.3 & 99.69 \\
\hline & $3 \mathrm{C}$ & $70-110$ & 44.37 & 24.07 & 4.36 & 1.70 & 2.47 & 0.55 & 2.87 & 0.89 & 0.26 & 0.04 & 18.1 & 99.67 \\
\hline & $4 \mathrm{C}$ & $110-125$ & 43.88 & 24.79 & 4.49 & 1.63 & 2.19 & 0.70 & 2.66 & 0.89 & 0.23 & 0.04 & 18.2 & 99.69 \\
\hline \multirow[t]{16}{*}{ LA2 } & OiC & $0-1$ & 38.57 & 18.13 & 9.55 & 1.62 & 2.38 & 0.32 & 1.89 & 0.68 & 0.48 & 0.09 & 26.0 & 99.70 \\
\hline & $\mathrm{AC} 1$ & $1-4$ & 47.10 & 21.26 & 13.98 & 2.20 & 3.30 & 0.37 & 2.19 & 0.81 & 0.58 & 0.13 & 7.7 & 99.64 \\
\hline & $\mathrm{AC} 2$ & $4-6$ & 33.54 & 11.86 & 43.93 & 1.39 & 1.37 & 0.20 & 1.08 & 0.49 & 0.35 & 0.17 & 5.3 & 99.73 \\
\hline & $\mathrm{C}$ & $6-25$ & 49.33 & 21.00 & 16.41 & 2.43 & 3.77 & 0.40 & 2.07 & 0.78 & 0.51 & 0.14 & 2.8 & 99.65 \\
\hline & $2 \mathrm{C}$ & $25-45$ & 52.13 & 22.48 & 12.82 & 2.46 & 3.26 & 0.50 & 2.30 & 0.83 & 0.39 & 0.13 & 2.3 & 99.64 \\
\hline & $3 \mathrm{C}$ & $45-65$ & 52.71 & 24.57 & 9.91 & 2.08 & 2.33 & 0.45 & 2.62 & 0.89 & 0.40 & 0.08 & 3.6 & 99.65 \\
\hline & $4 \mathrm{C}$ & $65-68$ & 52.24 & 25.89 & 9.31 & 2.15 & 2.08 & 0.47 & 2.71 & 0.98 & 0.37 & 0.07 & 3.3 & 99.63 \\
\hline & $5 \mathrm{C}$ & $68-73$ & 46.80 & 24.64 & 5.73 & 1.79 & 1.82 & 0.48 & 2.64 & 0.89 & 0.27 & 0.05 & 14.5 & 99.69 \\
\hline & $6 \mathrm{C}$ & $73-80$ & 38.81 & 22.06 & 3.90 & 1.41 & 1.69 & 0.42 & 2.21 & 0.82 & 0.23 & 0.03 & 28.1 & 99.72 \\
\hline & $7 \mathrm{C}$ & $80-108$ & 53.02 & 21.17 & 11.89 & 2.58 & 3.52 & 0.45 & 2.26 & 0.80 & 0.25 & 0.14 & 3.6 & 99.67 \\
\hline & $8 \mathrm{C}$ & $108-110$ & 53.32 & 26.91 & 7.01 & 2.55 & 2.76 & 0.66 & 2.88 & 1.02 & 0.26 & 0.10 & 2.2 & 99.64 \\
\hline & $9 \mathrm{C}$ & $110-120$ & 47.11 & 25.91 & 6.13 & 1.95 & 3.70 & 0.59 & 2.75 & 0.98 & 0.42 & 0.07 & 9.9 & 99.58 \\
\hline & $10 \mathrm{C}$ & $120-128$ & 49.18 & 26.55 & 6.77 & 2.07 & 2.96 & 0.53 & 2.80 & 0.95 & 0.42 & 0.07 & 7.3 & 99.63 \\
\hline & $11 \mathrm{C}$ & $128-153$ & 45.57 & 26.76 & 5.88 & 2.53 & 3.31 & 0.78 & 2.54 & 1.00 & 0.92 & 0.06 & 10.1 & 99.49 \\
\hline & $12 \mathrm{C}$ & $153-165$ & 50.57 & 27.36 & 4.54 & 2.11 & 1.54 & 0.56 & 3.07 & 1.03 & 0.39 & 0.05 & 8.4 & 99.62 \\
\hline & $13 \mathrm{C}$ & $165-170$ & 51.17 & 28.10 & 4.97 & 2.41 & 1.97 & 0.74 & 3.08 & 1.12 & 0.55 & 0.06 & 5.3 & 99.54 \\
\hline \multirow[t]{12}{*}{ LA3 } & OeiC & $0-2$ & 34.93 & 14.38 & 3.80 & 1.02 & 1.04 & 0.22 & 1.59 & 0.55 & 0.33 & 0.08 & 41.8 & 99.78 \\
\hline & $\mathrm{OaeC}$ & $2-6$ & 39.92 & 18.99 & 5.10 & 1.28 & 1.14 & 0.28 & 2.10 & 0.73 & 0.36 & 0.11 & 29.6 & 99.73 \\
\hline & $\mathrm{AC}$ & $6-15$ & 46.88 & 24.99 & 6.80 & 1.79 & 1.34 & 0.36 & 2.70 & 0.95 & 0.37 & 0.10 & 13.4 & 99.67 \\
\hline & $\mathrm{C}$ & $15-37$ & 48.57 & 26.16 & 6.20 & 2.00 & 1.67 & 0.37 & 2.85 & 1.00 & 0.39 & 0.07 & 10.3 & 99.66 \\
\hline & $2 \mathrm{C}$ & $37-57$ & 47.00 & 24.97 & 6.05 & 1.91 & 2.23 & 0.38 & 2.74 & 0.93 & 0.36 & 0.07 & 13.0 & 99.65 \\
\hline & $3 \mathrm{C}$ & $57-67$ & 48.87 & 25.23 & 6.82 & 1.97 & 2.06 & 0.33 & 2.76 & 0.92 & 0.32 & 0.08 & 10.3 & 99.66 \\
\hline & $4 \mathrm{C}$ & $67-78$ & 50.33 & 26.13 & 5.54 & 1.93 & 1.58 & 0.44 & 2.90 & 1.03 & 0.36 & 0.06 & 9.3 & 99.65 \\
\hline & $5 \mathrm{C}$ & 78-90 & 50.00 & 27.10 & 5.44 & 2.01 & 1.42 & 0.48 & 2.99 & 1.04 & 0.45 & 0.06 & 8.6 & 99.62 \\
\hline & $6 \mathrm{C}$ & $90-100$ & 49.56 & 26.76 & 5.90 & 1.85 & 1.56 & 0.30 & 2.83 & 0.99 & 0.35 & 0.06 & 9.5 & 99.70 \\
\hline & $7 \mathrm{C}$ & $100-107$ & 49.41 & 27.44 & 5.34 & 1.88 & 1.48 & 0.30 & 2.86 & 1.05 & 0.44 & 0.06 & 9.3 & 99.65 \\
\hline & $8 \mathrm{C}$ & $107-122$ & 50.24 & 27.80 & 5.62 & 2.17 & 2.08 & 0.47 & 3.03 & 1.10 & 0.48 & 0.06 & 6.5 & 99.61 \\
\hline & $9 \mathrm{C}$ & $122-130$ & 48.22 & 24.63 & 8.28 & 2.29 & 2.29 & 0.31 & 2.63 & 0.87 & 0.32 & 0.09 & 9.8 & 99.71 \\
\hline \multirow[t]{7}{*}{ LA4 } & Oei & $0-3$ & 27.11 & 11.23 & 4.74 & 1.08 & 1.92 & 0.21 & 1.33 & 0.57 & 0.47 & 0.57 & 50.4 & 99.68 \\
\hline & $\mathrm{A} 1$ & $3-10$ & 40.88 & 21.03 & 8.35 & 1.59 & 1.34 & 0.34 & 2.21 & 1.17 & 0.41 & 1.21 & 20.8 & 99.51 \\
\hline & A2 & $10-18$ & 46.02 & 24.87 & 9.20 & 2.17 & 1.92 & 0.42 & 2.57 & 0.97 & 0.45 & 0.22 & 10.7 & 99.51 \\
\hline & $\mathrm{AC}$ & $18-30$ & 46.68 & 24.93 & 8.57 & 2.38 & 2.64 & 0.41 & 2.47 & 0.94 & 0.45 & 0.11 & 9.9 & 99.52 \\
\hline & $\mathrm{C}$ & $30-70$ & 46.52 & 24.75 & 9.81 & 2.09 & 2.64 & 0.35 & 2.57 & 0.88 & 0.43 & 0.09 & 9.4 & 99.57 \\
\hline & $2 \mathrm{C}$ & $70-115$ & 45.09 & 25.11 & 8.32 & 2.21 & 2.72 & 0.43 & 2.48 & 0.92 & 0.52 & 0.09 & 11.6 & 99.52 \\
\hline & $3 \mathrm{C}$ & $115-135$ & 48.02 & 24.25 & 11.04 & 2.17 & 2.49 & 0.41 & 2.61 & 0.89 & 0.39 & 0.10 & 7.2 & 99.57 \\
\hline
\end{tabular}




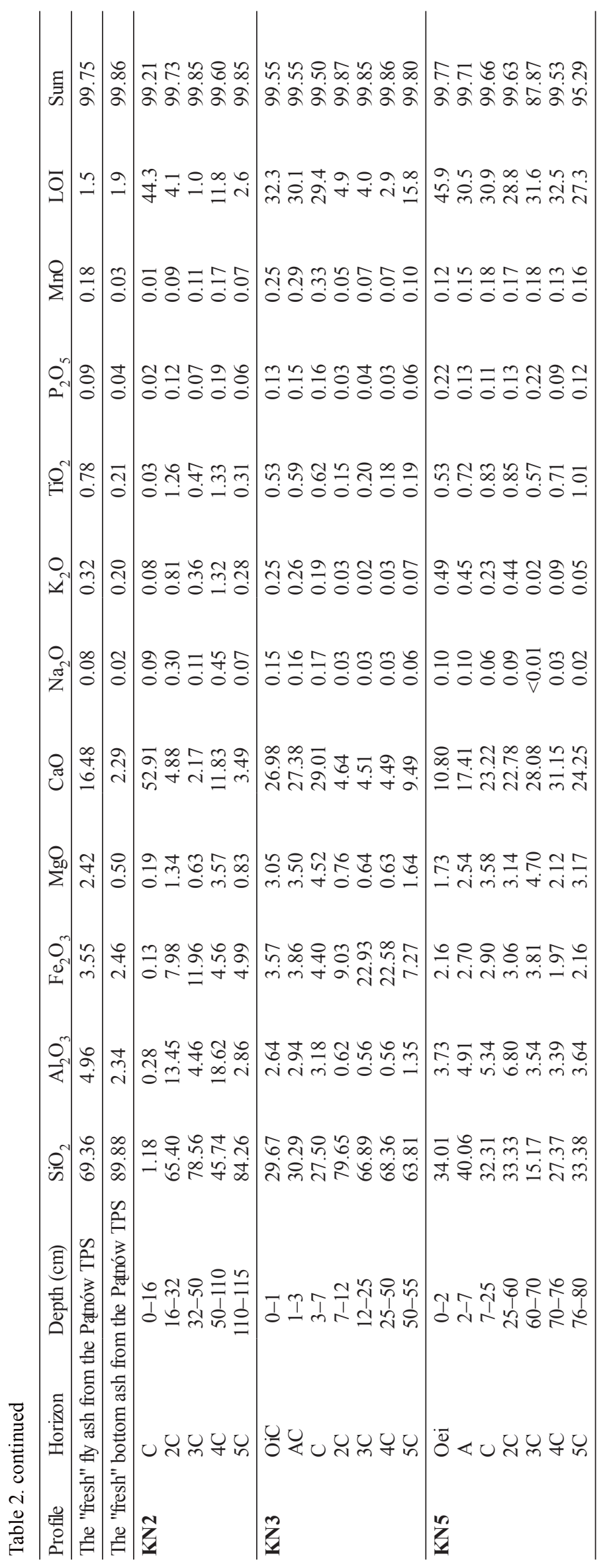

amounts of $\mathrm{Mn}(0.03-1.21 \%, 0.12 \%$ on average). LOI in "fresh" wastes after bituminous coal combustion was low (up to about 3\%), as they contained very little amounts of phases which were susceptible to burning (Table 2). However, in soils derived from these wastes LOI was diverse (from 2.2 to $50.4 \%, 13.1 \%$ on average). The highest LOI was found in organic horizons in which soil organic matter was volatilized after ignition of the sample. A high LOI in LA1 profile can be explained by high amounts of unburned organic fragments inherited from parent material.

Based on the chemical composition of "fresh" fly ash derived after bituminous coal combustion it can be classified as class F fly ash type (ASTM C618-15, 2015), as it fulfilled the following requirements: $\mathrm{SiO}_{2}+\mathrm{Al}_{2} \mathrm{O}_{3}+\mathrm{Fe}_{2} \mathrm{O}_{3}$ $\geq 70 \%$, LOI up to $5 \%$, up to $3 \%$ of $\mathrm{SO}_{3}$ (not shown), it contained alumino-silicate glass, and it had pozzolanic properties (i.e. siliceous and aluminous material which normally possesses little or no ability to cement, but which will have it after addition of water and calcium hydroxide) (Setina et al. 2013).

The chemical composition of the examined samples of wastes and soils derived after bituminous coal combustion was similar to that presented by Kierczak and Chudy (2014). On the other hand, the ashes derived after bituminous coal combustion examined in the present study (Table 2) contained more $\mathrm{Al}, \mathrm{Fe}, \mathrm{K}$, and $\mathrm{Na}$ in comparison with the ashes investigated by Maciak et al. (1974). These differences can be an effect of different methodology of major element analysis, or may result from the fact that the ashes derived after combustion of bituminous coals have initially different composition.

Silicon predominated in chemical composition of wastes and soils after lignite combustion (Table 2), as quartz was the major mineral in these samples (Table 1, Fig. 1C and D). Contents of $\mathrm{Si}$ were in the range $1.18-89.88 \%$ (48.39\% on average). The lowest amounts of Si were related to calcareous materials deposited in topsoil of the $\mathrm{KN} 2$ and $\mathrm{KN} 3$ profiles, and in some soil horizons in KN5 profile (Table 2). The second most abundant element in wastes and soils after lignite combustion was $\mathrm{Ca}$. Its amounts were between 2.17 and 52.91\% (17.06\% on average). The highest amounts of $\mathrm{Ca}$ were found in the samples in which $\mathrm{Ca}$ bearing minerals (carbonates, sulphates, oxides) occurred (Table 1), i.e. in the topsoil of the KN2 
and $\mathrm{KN} 3$ profiles, and in the subsoil of the $\mathrm{KN} 5$ profile. The wastes and soils after lignite combustion contained relatively high amounts of $\mathrm{Fe}$ (contents of 0.13 $22.93 \%, 6.10 \%$ on average) (Table 2). The lowest amounts of $\mathrm{Fe}$ were present in calcareous material, and the highest occurred in soil horizons rich in iron oxides (magnetite and hematite), e.g. in 3C and 4C horizons in $\mathrm{KN} 3$ profile (Table 1). The contents of $\mathrm{Al}$ in the wastes and soils discussed were between 0.28 and $18.62 \%$ (4.29\% on average). Lower amounts of $\mathrm{Al}$ in wastes and soils after lignite combustion in comparison with the wastes and soils after bituminous coal combustion can be related to lower contents or even lack of Al-bearing mineral phases (e.g. mullite or Al-bearing glass) in the former. The contents of $\mathrm{Mg}$ in the wastes and soils after lignite combustion ranged between 0.19 and $4.70 \%$ (2.15\% on average). Titanium was also present in these samples (contents of $0.03-1.33 \%, 0.57 \%$ on average). The wastes and soils after lignite combustion contained low amounts of K $(0.02-1.32 \%, 0.29 \%$ on average $)$, Mn $(0.01-$ $0.33 \%, 0.14 \%$ on average $), \mathrm{Na}(0.02-0.45 \%, 0.11 \%$ on average), and $\mathrm{P}(0.02-0.22 \%, 0.11 \%$ on average $)$ (Table 2). LOI in "fresh" wastes derived after lignite combustion was low, up to about $2 \%$. On the other hand, LOI in the soils built of material derived from wastes after lignite combustion ranged from 1.0 to $45.9 \%$ ( $21.6 \%$ on average) (Table 2 ). High LOI was found in these soil horizons which contained the highest amounts of soil organic matter (i.e. samples from the topsoils). Furthermore, high LOI was also found in soil horizons containing calcite (e.g. C horizon in KN2 profile, upper part of $\mathrm{KN} 3$ profile, and the whole KN5 profile), as this mineral was decomposed during the determination of LOI (i.e. during the ignition of soil samples at temperature of $\left.1000^{\circ} \mathrm{C}\right)$. Calcite decomposes at temperature $\sim 900^{\circ} \mathrm{C}$ (Bolewski 1982).

The chemical composition of "fresh" fly ash derived after lignite combustion indicates that it is similar to a class C fly ash type (ASTM C618-15, 2015). However, despite having cementitious properties (i.e. having the nature of cement) and content of lime $(\mathrm{CaO})$, it cannot be classified as typical class $\mathrm{C}$ fly ash, as the following criterion was not fulfilled: $\mathrm{SiO}_{2}+\mathrm{Al}_{2} \mathrm{O}_{3}+\mathrm{Fe}_{2} \mathrm{O}_{3} \geq 50 \%$.

The chemical composition of the "fresh" ashes and soil materials derived after lignite ashes shown in the present study (Table 2) is similar to that presented by Koukouzas et al. (2006). Moreover, contents of major elements in the KN5 profile developed on the landfill of the Konin TPS are comparable with the results by Maciak et al. (1974) obtained for the ashes from the Konin TPS and deposited most likely in the same landfill. However, for some reasons the ashes examined by Maciak et al. (1974) contained 3 times more Al and had 3 times lower LOI. Differences in Al contents between the samples studied in the present study (Table 2) and the samples of Maciak et al. (1974), may result from the fact that each portion of ash deposited in a landfill can differ from another in chemical composition, which is a common feature of ashes from TPSs. An increase of LOI can be explained by an increase of amounts of hydrated minerals (e.g. sulphates) in ashes several dozen of years after deposition on landfill of the Konin TPS.

\section{CONCLUSIONS}

1. There are clear differences between mineral and chemical composition of soils developed from wastes derived after combustion of bituminous coal and lignite. This is an effect of diverse mineral and chemical composition of "fresh" wastes.

2. The soils developed from wastes derived after combustion of bituminous coal consisted mainly of mineral phases inherited from "fresh" ashes (i.e. quartz, mullite, amorphous glass, and magnetite). However, relatively small amounts of calcite which was most likely a secondary (pedogenic) mineral, also occurred. Moreover, secondary iron oxides (goethite and lepidocrocite) resulting from the weathering of magnetite, were present in certain soil horizons.

3. The mineralogy of soils developed from wastes derived after combustion of lignite was more complex. "Fresh" fly ash after lignite combustion contained quartz as a predominating mineral, however anhydrite, lime, and iron oxides (magnetite and hematite) were also present. Quartz and iron oxides remained in the soils as inherited phases, however anhydrite and lime transformed into a variety of secondary sulphates (e.g. gypsum, bassanite, ettringite) and carbonates (calcite, vaterite). Calcareous materials deposited in the topsoil of some profiles consisted mainly of calcite.

4. The major elements in the samples (i.e. "fresh" ashes and soil samples) derived after bituminous coal combustion can be arranged in a following order based on average contents (from the most to the least abundant): $\mathrm{Si}>\mathrm{Al}>\mathrm{Fe}>\mathrm{K}>\mathrm{Ca}>\mathrm{Mg}>\mathrm{Ti}>\mathrm{Na}$ $>\mathrm{P}>\mathrm{Mn}$. On the other hand, the abundances of major elements in the samples after lignite combustion are as follows: $\mathrm{Si}>\mathrm{Ca}>\mathrm{Fe}>\mathrm{Al}>\mathrm{Mg}>\mathrm{Ti}>\mathrm{K}>\mathrm{Mn}$ $>\mathrm{Na}>\mathrm{P}$. However, it should be noted that in some horizons (i.e. in calcareous materials deposited in the topsoil of some profiles) in soils developed from wastes derived after lignite combustion, $\mathrm{Ca}$ was a clearly predominating element followed by lower amounts of other major elements. 


\section{ACKNOWLEDGMENTS}

Research funded by the Polish National Science Centre (decision no. DEC-2011/03/D/ST10/04599). Mrs. Agnieszka Ostrowska (the Analytical Centre, Warsaw University of Life Sciences - SGGW) is acknowledged for her assistance during the SEM observations. The authors would like to thank the reviewer for constructive comments and suggestions.

\section{REFERENCES}

ASTM (American Society for Testing and Materials) C618-15, 2015. Standard Specification for Coal Fly Ash and Raw or Calcined Natural Pozzolan for Use in Concrete. Book of Standards Volume: 04.02. ASTM International, West Conshohocken, PA.

Bolewski A., 1982. Mineralogia szczegółowa. $3^{\text {rd }}$ editon. Wydawnictwa Geologiczne, Warszawa: pp. 542 (in Polish).

IUSS Working Group WRB, 2015. World Reference Base for Soil Resources 2014, update 2015. International soil classification system for naming soils and creating legends for soil maps. World Soil Resources Reports No. 106. FAO, Rome: pp. 192.

Kierczak J., Chudy K., 2014. Mineralogical, chemical, and leaching characteristics of coal combustion bottom ash from a power plant located in northern Poland. Polish Journal of Environmental Studies, 23(5): 1627-1636.

Koukouzas N.K., Zeng R., Perdikatsis V., Xu W., Kakaras E.K., 2006. Mineralogy and geochemistry of Greek and Chinese coal fly ash. Fuel, 85: 2301-2309.

Kutchko B.G., KimA.G., 2006. Fly ash characterization by SEM-EDS. Fuel, 85: 2537-2544.

Maciak F., Liwski S., Biernacka E., 1974. Some physico-chemical and biochemical properties of the ash dumps after brown and hard coal. Roczniki Gleboznawcze - Soil Science Annual, 25(3): 191-205 (in Polish with English summary).
Maciak F., Liwski S., Biernacka E., 1976. Agricultural recultivation of furnace waste (ash) dumps from brown and hard coal. Part III. Course of soil-forming processes in ash dumps under influence of grass and leguminous vegetation. Roczniki Gleboznawcze - Soil Science Annual, 27(4): 189-209 (in Polish with English summary).

Pansu M., Gautheyrou J., 2006. Handbook of Soil Analysis. Mineralogical, Organic and Inorganic Methods. Springer-Verlag, Berlin Heidelberg: pp. 993.

Rodriguez-Navarro C., Jimenez-Lopez C., Rodriguez-Navarro A., Gonzalez-Muńoz M.T., Rodriguez-Gallego M., 2007. Bacterially mediated mineralization of vaterite. Geochimica et $\mathrm{Co}-$ smochimica Acta, 71(5): 1197-1213.

Rowlands D.L.G., Webster R.K., 1971. Precipitation of vaterite in lake water. Nature Physical Science, 229: 158-158.

Setina J., Gabrene A., Juhnevica I., 2013. Effect of pozzolanic additives on structure and chemical durability of concrete. Procedia Engineering, 57: 1005-1012.

Sokol E.V., Maksimova N.V., Volkova N.I., Nigmatulina E.N., Frenkel A.E., 2000. Hollow silicate microspheres from fly ashes of the Chelyabinsk brown coals (South Urals, Russia). Fuel Processing Technology, 67: 35-52.

Sokol E.V., Kalugin V.M., Nigmatulina E.N., Volkova N.I., Frenkel A.E., Maksimova N.V. 2002. Ferrospheres from fly ashes of Chelyabinsk coals: Chemical composition, morphology and formation conditions. Fuel, 81: 867-876.

Thomas M., Folliard K., Drimalas T., Ramlochan T., 2008. Diagnosing delayed ettringite formation in concrete structures. Cement and Concrete Research, 38(6): 841-847.

Warren C.J., Dudas M.J., 1985. Formation of secondary minerals in artificially weathered fly ash. Journal of Environmental Quality, 14: 405-410.

Yeheyis M.B., Shang J.Q., Yanful E.K., 2009. Chemical and mineralogical transformations of coal fly ash after landfilling. Proceedings of the World of Coal Ash Conference, Lexington, KY, USA: 1-13.

Zikeli S., Jahn R., Kastler M., 2002. Initial soil development in lignite ash landfills and settling ponds in Saxony-Anhalt, Germany. Journal of Plant Nutrition and Soil Science, 165: $530-536$

Received: December 7, 2015

Accepted: December 30, 2015

\section{Skład mineralny i chemiczny technogenicznych utworów glebowych (Technosols) wytworzonych $\mathrm{z}$ odpadów popiołowo-żużlowych z wybranych polskich elektrowni węglowych}

\footnotetext{
Streszczenie: Celem przeprowadzonych badań było określenie składu mineralnego i chemicznego technogenicznych utworów glebowych (Technosols) wytworzonych z odpadów popiołowo-żużlowych pochodzących z elektrowni węglowych spalających węgiel brunatny i kamienny. Skład mineralny próbek glebowych oraz ,świeżych” popiołów lotnych i żużli badano metodą dyfrakcji rentgenowskiej (XRD) oraz za pomocą skaningowego mikroskopu elektronowego (SEM). Skład chemiczny (zawartość pierwiastków głównych) badano metodą ICP-AES. W składzie mineralnym „świeżych” popiołów lotnych i żużli po spaleniu węgla kamiennego dominował kwarc, mullit oraz mineralna substancja amorficzna (szkliwo). Występowały tam również tlenki żelaza (głównie magnetyt). Utwory glebowe wytworzone z popioło-żużli po spaleniu węgla kamiennego zawierały wymienione wyżej minerały odziedziczone z popiołów i żużli. Oprócz nich występowały w utworach glebowych stosunkowo niewielkie ilości wtórnego kalcytu. W niektórych poziomach glebowych zawierających szczególnie duże ilości odziedziczonego magnetytu, występowały również wtórne tlenki i tlenowodorotlenki żelaza (getyt i lepidokrokit). W składzie mineralnym „świeżych” popiołów lotnych i żużli po spaleniu
} 
węgla brunatnego dominował kwarc, występowały tam również stosunkowo małe ilości tlenków żelaza (magnetyt i hematyt). W popiołach lotnych, oprócz wyżej wymienionych minerałów, zidentyfikowano ponadto anhydryt oraz tlenek wapnia. Utwory glebowe wytworzone z popioło-żużli po spaleniu węgla brunatnego zawierały kwarc, magnetyt i hematyt odziedziczone z popiołów i żużli. Ponadto w utworach tych występował kalcyt, który w niektórych warstwach glebowych był minerałem dominującym. W utworach glebowych obecne były również siarczany (gips, bassanit i ettringit), a także vateryt - rzadko spotykana odmiana polimorficzna węglanu wapnia. W składzie chemicznym popiołów i gleb wytworzonych z odpadów po spaleniu węgla kamiennego dominował Si. Pozostałe pierwiastki główne można uszeregować następująco według ich malejących zawartości: Al, $\mathrm{Fe}, \mathrm{K}, \mathrm{Ca}, \mathrm{Mg}, \mathrm{Ti}, \mathrm{Na}$, $\mathrm{P}$ i Mn. Natomiast pierwiastki główne w próbkach po spaleniu węgla brunatnego można uszeregować malejąco w następująca sekwencję: $\mathrm{Si}, \mathrm{Ca}, \mathrm{Fe}, \mathrm{Al}, \mathrm{Mg}, \mathrm{Ti}, \mathrm{K}, \mathrm{Mn}, \mathrm{Na}$ i P. Jednakże w niektórych poziomach wierzchnich zbudowanych z materiału węglanowego dominującym pierwiastkiem był $\mathrm{Ca}$, a pozostałe pierwiastki główne były obecne w mniejszych ilościach.

Słowa kluczowe: popiół lotny, żużel paleniskowy, Technosols, dyfrakcja rentgenowska, elektronowa mikroskopia skaningowa, pierwiastki główne 